patients and also by respiratory muscle limitation in $10 \%$ of the patients.

Conclusions: Based on admission diagnosis of 40 patients, $90 \%$ were defined as subject to pulmonary rehabilitation and in $10 \%$ were receiving cardiac rehabilitation. They divided into three specific functional recovery programs: ventilatory function recovery program $(60 \%)$, cardiac function recovery program $(30 \%)$, respiratory muscles recovery program $(10 \%)$.

\section{Leg Muscle Blood Flow During 1 and 2-Leg Knee Extension Exercise in Patients with COPD and Aged-Controls}

Robillard Julie, Fatihat Momoh, Jacinthe Baril, Montreal, Quebec, Canada

Andrew William Sheel, Vancouver, British-Columbia, Canada

Robert Boushel, David Laurin, Amanda Rossi, Julie Deschênes, Jean Bourbeau, Tanja Taivassalo, Hélène Perrault, Montreal, Quebec, Canada

Background: In chronic obstructive pulmonary disease (COPD), leg muscle blood flow may be compromised during dynamic exercise due to the competing influence of respiratory muscle work for available cardiac output. This study examined the flow demand limits of skeletal muscle flow in varying muscle mass recruitment. It employed one leg knee extension (1L$\mathrm{KE})$ and two-leg knee extension (2L-KE) to examine the mass-specific work rates at which peripheral circulatory function may become compromised due to elevated respiratory demands.

Methods: Three male COPD patients $(70 \pm 5 \mathrm{yr}$; $\left.\mathrm{FEV}_{1} / \mathrm{FVC}=42 \pm 11 \%\right)$ and two aged-controls (74 \pm $1 \mathrm{yr} ; \mathrm{FEV}_{1} / \mathrm{FVC}=76 \pm 5 \%$ ) completed three sets of 7-minute steady state $1 \mathrm{~L}-\mathrm{KE}$ and $2 \mathrm{~L}-\mathrm{KE}$ at 20, 40 and $65 \%$ (SS20\%; SS40\%; SS65\%) of previously determined ergometer-specific peak power, separated by rest periods of $15 \mathrm{~min}$. Leg muscle blood flow (BF) was determined using pulsed Doppler sonography of the femoral artery during incremental exercise loads and post-exercise. ECG, blood pressure, ventilatory parameters and VO2 were obtained continuously, and dye dilution cardiac output was measured at rest and during exercise.

Results: Preliminary data showed that, for each exercise intensity, the required $\mathrm{VO}_{2}$ is similar in both groups. However, the workloads in COPD are between $60 \%$ and $82 \%$ of the control group workloads. During $1 \mathrm{~L}-\mathrm{KE}$ and $2 \mathrm{~L}-\mathrm{KE}, \mathrm{BF}$ is consistently higher in COPD compared to controls. For $1 \mathrm{~L}-\mathrm{KE}$, the increase in $\mathrm{BF}$ from rest (mean $\pm \mathrm{SD}$ in $\mathrm{ml} / \mathrm{min}$; COPD vs controls) are SS20\%: $763 \pm 244$ vs $105 \pm 34$; SS40\%: $1157 \pm 597$ vs $310 \pm 97 ;$ SS65\%: $1493 \pm 348$ vs $424 \pm 45$. BF relative to workload is at least 3 -fold higher in COPD compared to controls for all exercise intensities.

Conclusions: These data suggest that mean muscle blood flow may not be compromised during kneeextensor exercise in COPD patients, and ongoing data will clarify whether this is a compensatory response to altered peripheral muscle metabolic function.

\section{A Written Action Plan for Early Treatment of COPD Exacerbations: An Important Component to the Reduction of Hospitalizations}

Sedeno Maria F, MM, Respiratory Epidemiology and Clinical Research Unit, Montreal Chest Institute, McGill University, Montréal, Québec, Canada Diane Nault, MSc, Respiratory Epidemiology and Clinical Research Unit, Montreal Chest Institute, McGill University and Chronic Obstructive Pulmonary Disease axis of the Respiratory Network, Fonds de la Recherche en Santé du Québec (FRSQ), Montréal, Québec, Canada

Dina H. Hamd, PhD, Respiratory Epidemiology and Clinical Research Unit, Montreal Chest Institute, McGill University, Montréal, Québec, Canada Jean Bourbeau, MD, Respiratory Epidemiology and Clinical Research Unit, Montreal Chest Institute, McGill University and Chronic Obstructive Pulmonary Disease axis of the Respiratory Network, Fonds de la Recherche en Santé du Québec (FRSQ), Montréal, Québec, Canada

Background: This study explored the role of a written action plan in reducing hospitalizations as a com- 